\title{
Monte Carlo Simulations of Million Body Star Clusters
}

\author{
Mirek Giersz \\ Nicolaus Copernicus Astronomical Center Polish Academy of Sciences, \\ ul. Bartycka 18, 00-716 Warsaw, Poland
}

\begin{abstract}
The first results of Monte Carlo simulations of the evolution of tidally limited, multi-mass million star clusters with stellar evolution and dynamically formed binaries are presented.
\end{abstract}

\section{Method and initial models}

A revision of Stółkiewicz's Monte Carlo code (Stółkiewicz 1982, 1986, Giersz 1998,2001 ) is for the first time used to simulate the evolution of tidally limited, multi-mass million star clusters. The initial models are described by the King models with $W_{0}=5,7$, Salpeter's initial mass function with masses in the range of $0.1 M_{\odot}-15 M_{\odot}$. The total system mass is $319305 M_{\odot}$ and the tidal radius is 33.57 pc. Stellar evolution is described according to Chernoff \& Weinberg (1990). The process of energy generation is realized by means of appropriately modified versions of Spitzer's and Mikkola's formulae for the interaction cross section between binaries and field stars and binaries themselves (Spitzer 1987 and reference therein). There are no primordial binaries.

\section{Results}

The evolution of anisotropy strongly depends on the initial system concentration. For less concentrated systems, very strong initial mass loss due to stellar evolution forces anisotropy in the middle and outer parts of the system to become negative. It stays negative until the time close to cluster disruption, after which it becomes slightly positive. For systems initially strongly concentrated, at the beginning anisotropy behaves like in isolated systems. Positive anisotropy develops throughout most of the system, which is connected with weaker initial mass loss. When tidal stripping becomes important, anisotropy is reduced, and in the outer parts of the system become's negative.

The evolution of the global cluster mass function is very similar to the evolution of the mass function in the middle parts of the system. Generally, the mass function is shallower in the central parts of the system and steeper in the outer parts. In the course of evolution the mass function becomes shallower in every part of the system. This reflects the fact of stellar evolution (destruction of the most massive stars), mass segregation and tidal stripping (removal of less massive stars).

During the late phases of evolution clusters contain a substantial population of evolved stars (up to $50 \%$ and even more, close to the disruption time). The 


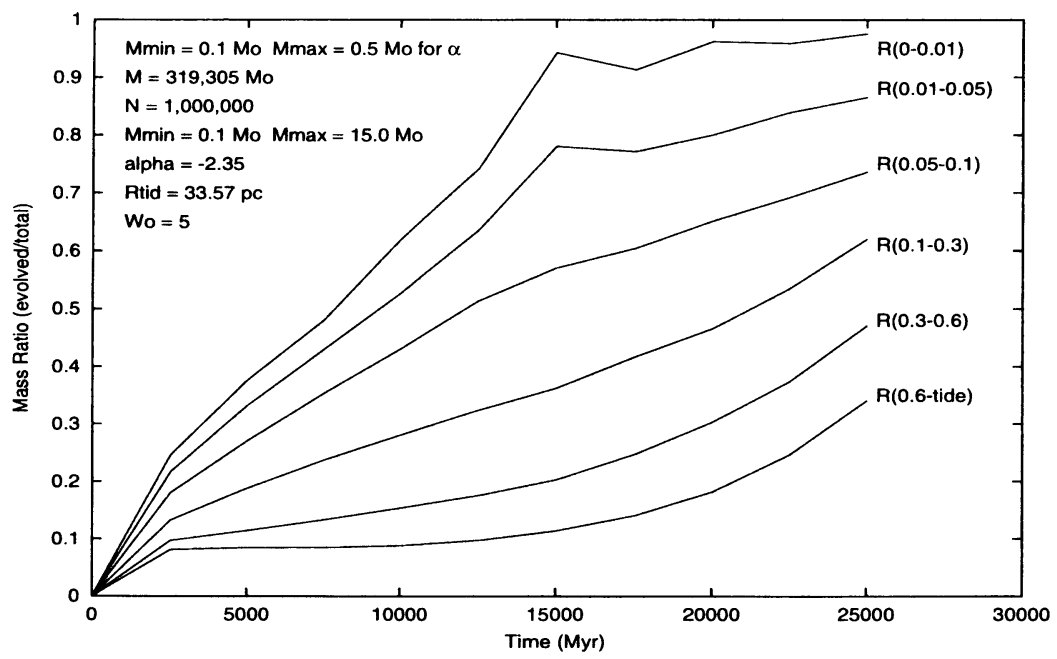

Figure 1. Evolution of the mass ratios of the evolved stars to the total mass for different Lagrangian shells: $0 \%-1 \%, 1 \%-5 \%, 5 \%-$ $10 \%, 10 \%-30 \%, 30 \%-60 \%$, and $60 \%$ - tidal radius for model $\mathrm{W} 5$.

cluster core (about $0.5 \%$ Lagrangian radius) is mostly populated by evolved stars, only less than $5 \%$ by mass are main sequence stars (see Fig. 1). Even at the outer parts of the system evolved stars are very abundant. The number of abundances of different species of stars in the system show the same picture. The core consists of more than $90 \%$ (by number) evolved stars and the outer parts of the system up to $10 \%$, respectively.

\section{Conclusions}

The results presented here show that the Monte Carlo method is a robust scheme to study, in an effective way, the evolution of large $N$-body systems. It provides, in almost as much detail as direct $N$-body simulations, information about the movement of any object in the system. The first results suggest that real globular clusters (orbiting the Milky Way) could contain a very substantial amount of unseen mass in the form of old white dwarfs and neutron stars/black holes.

\section{References}

Chernoff, D.F. \& Weinberg, M.D. 1990, ApJ, 351, 121

Giersz, M. 1998, (Paper I), MNRAS, 298, 1239

Giersz, M. 2001, (Paper II), MNRAS, 324, 218

Spitzer, L. 1987, in Dynamical Evolution of Globular Clusters, Princeton University Press, Princeton

Stdółkiewicz, J.S. 1982, Acta Astronomica, 32, 63

Stdółkiewicz, J.S. 1986, Acta Astronomica, 36, 19 